\title{
A study of refractory cases of persistent epithelial defects associated with dry eye syndrome and recurrent corneal erosions successfully treated with cyclosporine A $0.05 \%$ eye drops
}

This article was published in the following Dove Press journal:

Drug Design, Development and Therapy

\author{
Pietro Emanuele \\ Napoli (D) ${ }^{1, *}$ \\ Mirco Braghiroli (D) ${ }^{1, *}$ \\ Claudio lovino (D) \\ Giuseppe Demarinis (ID) \\ Maurizio Fossarello (iD) ${ }^{1,2}$ \\ 'Department of Surgical Sciences, Eye \\ Clinic, University of Cagliari, Cagliari, \\ Italy; ${ }^{2}$ Clinica Oculistica, San Giovanni di \\ Dio Hospital, Azienda Ospedaliera \\ Universitaria di Cagliari, Cagliari, Italy \\ *These authors contributed equally to \\ this work
}

Background: Effective and tolerable therapeutic strategies for patients with refractory persistent epithelial defects (PEDs) are limited and generally provide poor outcomes. This retrospective case review describes four refractory cases of PEDs associated with recurrent corneal erosions (RCEs) and dry eye disease (DED), which were successfully treated with cyclosporine eye drops.

Methods: Patients were treated with cyclosporine A $0.05 \%$ eye drops twice a day for at least 12 months. At enrolling time, each patient was asked to suspend topical steroids or other eye drops used for ocular surface abnormalities with the exception of lubricants and eye washing. A complete evaluation of ocular surface symptoms was performed including the McMonnies Dry Eye Questionnaire, Ocular Surface Disease Index, slit-lamp biomicroscopy, fluorescein break-up time, the fluorescein staining of the cornea and conjunctiva (according to the Oxford grading system), the Schirmer I test, and the meibomian secretion after digital pressure application on the lids. This set of exams was carried out at baseline and repeated at all follow-up assessments.

Results: All participants that did not benefit from previous therapies, including corticosteroids tapering schedule, showed an important improvement in the clinical picture after two months with topical cyclosporine medication. Moreover, after 12 months of continuous therapy, all patients showed a clinical improvement in DED signs and symptoms, related to the absence of new RCE episodes. The treatment was well tolerated, and no adverse effects were reported.

Conclusion: Although a small number of cases were available of our analysis, the treatment with cyclosporine eye drops represents a promising approach in the management of refractory PEDs with associated ocular comorbidities, since it may reduce the RCE episodes and improve the tear film stability, in absence of systemic or local side effects.

Keywords: dry eye disease, persistent epithelial defects, cyclosporine eye drops, recurrentcorneal erosion, corneal adhesion

\section{Introduction}

A healthy corneal epithelium is fundamental to prevent damages or infections of the deeper ocular structures, in order to permit, in conjunction with the tear film, a normal vision. ${ }^{1,2}$ Accordingly, several corneal epithelial diseases may lead to devastating consequences to the overall ocular health. ${ }^{3,4}$ In this sense, corneal epithelial defects are focal areas of epithelial loss, and therefore local regions of minor defenses of the ocular surface. ${ }^{5}$
Correspondence: Pietro Emanuele Napoli Department of Surgical Sciences, Eye Clinic, University of Cagliari, Via

Ospedale 46, Cagliari 09124, Italy Tel +393208643697

Email pietronapoli@ymail.com 
Clinically, epithelial defects may be long lasting when the alterations remain at least two weeks. In these cases, they are named "Persistent Epithelial Defects" (PEDs). ${ }^{6}$ Areas of epithelial loss can be easily revealed by fluorescein dye during biomicroscopic evaluation. ${ }^{5}$

A variable number of PEDs may lead to recurrent, painful episodes of breakdown of corneal epithelium that are defined as recurrent corneal erosions (RCEs). Each episode of RCE is characterized by the sudden onset of eye pain, redness, and photophobia, usually at night or upon awakening. RCE episodes could last from hours to days. ${ }^{7}$ There are different causes of PEDs, such as epithelial/limbal stem cell deficiency, inflammatory diseases, neurotrophic diseases (common causes include diabetes, herpetic infection, and nerve damage from ocular surgery), and mechanical factors, especially abnormal lid pathologies. ${ }^{1}$

On the other hand, dry eye disease (DED) is a set of disorders of the ocular surface, which can lead to ocular discomfort, pain, blurred vision, and other symptoms and signs. DED represents a potential, mechanical, and inflammatory cause of PEDs. In 2017, the International Dry Eye WorkShop (DEWS) defined DED as a "multifactorial disease of the ocular surface" defined by a loss of homeostasis of the tear film with ocular surface inflammation and damage, as well as neurosensory abnormalities. ${ }^{8}$ DED may be episodic or chronic. In particular, when the disease becomes chronic and severe, if untreated, patients may even develop ocular surface damage. ${ }^{9}$

Although there are several treatment strategies for PEDs, no topical treatment demonstrated to be overall effective for ocular surface damage, ocular dryness, and the recurrent breakdown of the epithelium.

Topical cyclosporine A $0.05 \%$ is an immunomodulatory agent that has been shown to reduce chronic ocular surface inflammation by decreasing the cell-mediated inflammatory responses. For these reasons, it has been approved for the treatment of DED by the US Food and Drug Administration (FDA) since April 2003, but its potential in other ocular surface diseases is still poorly understood.

In this case series, we topically administered cyclosporine A $0.05 \%$ in patients with PEDs associated with DED and RCEs to assess its potential role on the ocular surface healing and in reducing the recurrence rate of corneal erosions.

\section{Materials and methods}

\section{Subjects and study procedure}

This descriptive, retrospective study was conducted at the Eye Clinic of the Department of Surgical Sciences,
University of Cagliari, Italy. The study adhered to the tenets of the Declaration of Helsinki and was approved by the Office of Research Ethics, University of Cagliari. All subjects signed informed consents prior to their participation in the study.

Four consecutive patients (one male and three females) suffering from ocular discomfort symptoms attributable to PEDs associated with DED and RCEs were referred to our Eye Clinic from January 2015 to December 2017.

Patients were examined at baseline and on the 15th day, and on the 2nd, 6th, and 12th month after the onset of the therapy.

Inclusion criteria were as follows: recalcitrant PEDs, DED with ocular discomfort symptoms (ie, dryness, soreness, scratchiness, burning, and grittiness), and history of RCEs (presenting by pain, redness, and photophobia). Exclusion criteria included conjunctivitis or different active ocular inflammation or infection.

At enrolling time, each patient was asked to suspend topical steroids or other eye drops used for ocular surface abnormalities with the exception of lubricants and eye washing. Thus, patients were treated with cyclosporine A $0.05 \%$ eye drops twice a day for at least 12 months.

\section{Patients' medical history and clinical picture \\ Patient $\mathrm{I}$ and 2}

Two of the study patients were women, aged 56 and 64 years, who complained about DED symptoms for five and two years, respectively. They also referred monocular recurrent episodes of pain, redness, and photophobia (at least one episode per month) usually starting on the first awakening. In the months before the first visit, patients consulted other ophthalmologists, thus receiving topical eye drops such lubricants and steroids, with temporary and partial improvement of ocular symptoms. At the slitlamp evaluation, they showed multiple PEDs in one eye. General anamnesis was unremarkable in both cases.

\section{Patient 3}

The third patient was a 25 -year-old woman, with a long history of DED (for at least 6 years) unsuccessfully treated with ocular lubricants and topical steroids. Clinically, she showed a PED in the lower quadrant of the right eye. She also referred recurrent episodes of acute pain (more than three times per month) in the left eye due to RCEs episodes despite the use of bandage contact lens. During anamnesis, patient reported suffering type 1 diabetes 
mellitus (T1DM), anemia, numerous food allergies, and autoantibody positivity (ANA and ANCA).

\section{Patient 4}

The fourth patient was a 41-year-old man, who developed a PED in the left eye after herpes simplex keratitis. For about two years, he has been complaining of monthly episodes of pain, photophobia, and redness in the right eye. He was treated with inhibitors of matrix metalloproteinase-9 (oral doxycycline $50 \mathrm{mg}$ two times daily) associated with lubricant eye drops, topical steroids, and eye washing without substantial benefits. Clinically, he presented a single PED in the left eye associated with a mild form of DED. General anamnesis was unremarkable.

\section{Clinical assessment}

At the first evaluation, the data collected included the best corrected visual acuity (BCVA) expressed in logMAR score, the intraocular pressure (IOP) determined using Goldmann tonometer, and general/ocular anamnesis. (Table 1). Medical records of patients were reviewed retrospectively.

On clinical assessment, a complete evaluation of ocular surface signs and symptoms was performed as reported in previous studies under controlled environmental conditions. ${ }^{10-12}$ In particular, a complete evaluation of ocular surface symptoms was performed using McMonnies Dry Eye Questionnaire and Ocular Surface Disease Index (OSDI). These questionnaires provide a score that represents the degree of severity of the DED condition or symptoms. With regard to the McMonnies score, the classification of relative DED grade is as follows: if score is less than 10, it means no DED; between 10 and 20, DED is mild; more than 20, DED is considered severe. With respect to the OSDI score, relative DED grade classification is as follows: if score is less than 12 , it means no DED; between 13 and 22, DED is mild; between 23 and 32, DED is moderate; more than 33, DED is considered severe.

In addition, the ocular surface was analyzed using by slit-lamp biomicroscopy (Figures 1 and 2). After the instillation of fluorescein dye in the tear film (blue cobalt light with yellow filter Wratten n.12), we evaluated the fluorescein break-up time (FBUT), the fluorescein staining of the cornea and conjunctiva (according to the Oxford grading system), the Schirmer I test, and the meibomian secretion (meibum) after digital pressure application on the lids. ${ }^{13-15}$

Table I Baseline data

\begin{tabular}{|c|c|c|c|c|}
\hline & Patient I & Patient 2 & Patient 3 & Patient 4 \\
\hline Sex & Female & Female & Female & Male \\
\hline Age (years) & 56 & 64 & 25 & 41 \\
\hline Ocular anamnesis & $\begin{array}{l}\text { DED, PEDs } \\
\text { and RCEs }\end{array}$ & $\begin{array}{l}\text { DED, PEDs } \\
\text { and RCEs }\end{array}$ & DED, PEDs and RCEs & $\begin{array}{l}\text { DED, PEDs and RCEs after } \\
\text { herpes simplex keratitis in L.E. }\end{array}$ \\
\hline General anamnesis & Unremarkable & Unremarkable & $\begin{array}{l}\text { TIDM, anemia, food allergies and autoan- } \\
\text { tibodies positivity. }\end{array}$ & Unremarkable \\
\hline Affected eye (PEDs/RCEs) & Right & Left & Right & Left \\
\hline BCVA R.E- L.E (logMAR) & $0.00-0.00$ & $0.10-0.10$ & $0.00-0.00$ & $0.00-0.00$ \\
\hline IOP R.E.- L.E.(mmHg) & $14-15$ & $18-20$ & $17-16$ & $19-18$ \\
\hline McM (score) & 15 & 17 & 29 & 16 \\
\hline OSDI (score) & 30.5 & 33.3 & 70.8 & 30.2 \\
\hline FBUT R.E. (s) & 4 & 3 & 3 & 4 \\
\hline FBUT L.E. (s) & 3 & 2 & 3 & 5 \\
\hline Oxford R.E (grade) & 0 & 1 & 0 & 0 \\
\hline Oxford L.E. (grade) & 0 & 2 & 0 & 0 \\
\hline Schir. R.E. (mm) & 2 & 1 & 4 & 7 \\
\hline Schir. L.E. (mm) & I & 0 & 6 & 7 \\
\hline Meib. R.E. (grade) & Normal & Normal & Poor & Normal \\
\hline Meib. L.E. (grade) & Normal & Normal & Poor & Normal \\
\hline RCEs episodes (rate) & I per month & I per month & $>3$ per month & I per month \\
\hline
\end{tabular}

Abbreviations: PEDs, persistent epithelial defects; DED, dry eye disease; RCEs, recurrent corneal erosions; HSV, herpes simplex virus; TIDM, type I diabetes mellitus; BCVA, best corrected visual acuity; McM, McMonnies Dry Eye Questionnaire (score); OSDI, Ocular Surface Disease Index (score); FBUT R.E/L.E., fluorescein break-up time in right eye/left eye expressed in seconds; Oxford R.E/L.E., fluorescein staining of the cornea and conjunctiva (according to the Oxford grading system) in right eye/left eye; Schir. R.E/L.E, Schirmer I test in the right/left eye (expressed in mm); Meib. R.E/L.E., meibomian secretion (meibum) after digital pressure application on the upper lid tarsus; RCEs episodes, presence of episodic RCEs symptoms (acute pain and photophobia). 


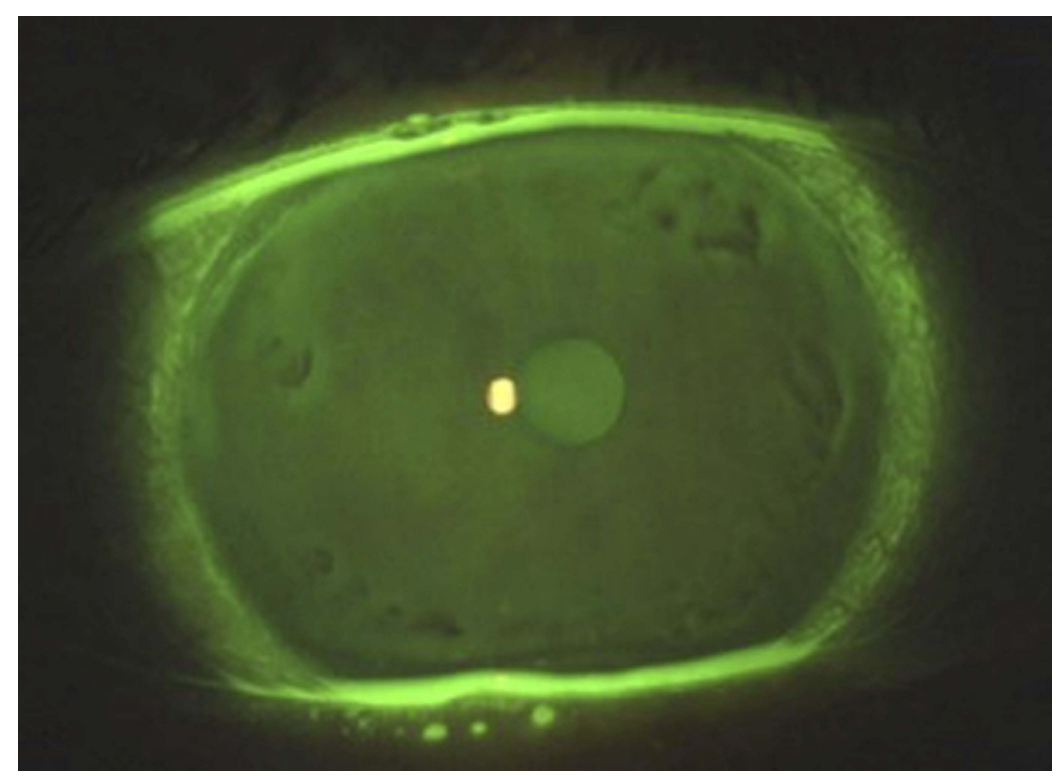

Figure I Ocular surface with epithelial abnormalities. After the instillation of fluorescein dye in the tear film (blue cobalt light with yellow filter written n. I2), the ocular surface revealed multiple patterns of epithelial abnormalities, which are seen as non-colored areas of the tear film (located at the same site during the various blinking). Clearly, the latter represent irregularities of the epithelial-basal membrane complex. When recurrent erosions occur, the site of epithelial defects corresponds to those of the epithelial blisters. If an epithelial defect lasts more than two weeks, it can be considered as "persistent" (or PED).

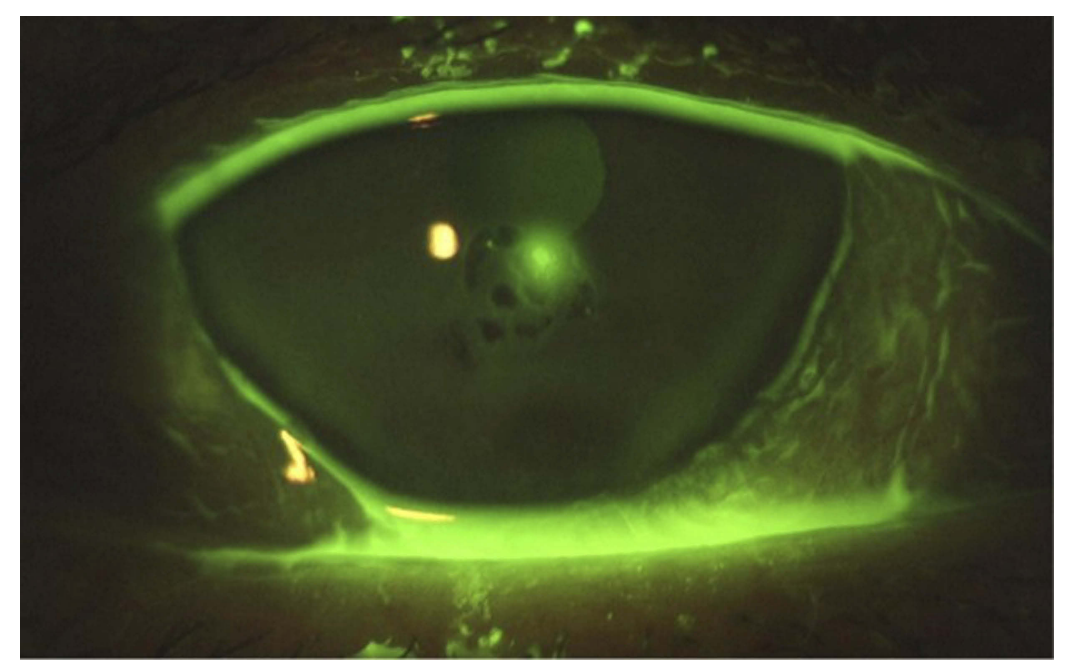

Figure 2 Ocular surface with a persistent epithelial defect (PED). Epithelial defects are considered as "persistent" when they last at least 2 weeks without regression. In this image, after the instillation of fluorescein dye in the tear film (blue cobalt light with yellow filter Written $\mathrm{n}$. 12), this patient clearly showed a paracentral area of epithelial loss, which was refractory to the first-line treatment.

This set of exams was repeated in each visit during the follow-up period of 12 months (Table 2). Each examination was completed within 25-30 mins and in the same day time (from $3 \mathrm{pm}$ to $5 \mathrm{pm}$ ).

\section{Results}

Overall, the mean patient age at the first evaluation was 46.5 years (range 25 to 64 ).

\section{Baseline}

All patients presented to our attention were complaining DED symptoms (ie, dryness, soreness, scratchiness, burning, and grittiness) in both eyes for at least two years. Beyond the DED symptoms, our patients claimed recurrent episodes of pain, redness, and photophobia (RCEs) in the eye affected by PEDs. These painful episodes usually started in the morning, on the first awakening, and lasted about two or three days. 
Table 2 Results during therapy

\begin{tabular}{|c|c|c|c|c|c|c|c|c|c|c|c|c|c|}
\hline \multirow{2}{*}{\multicolumn{2}{|c|}{$\begin{array}{l}\text { Time after cyclospor- } \\
\text { ine A } 0.05 \% \text { eye drops } \\
\text { onset }\end{array}$}} & \multirow{3}{*}{$\begin{array}{l}\text { Eye with PEDs } \\
\text { Right }\end{array}$} & \multirow{3}{*}{$\begin{array}{l}\mathbf{M c M} \\
15 \\
13 \\
8 \\
10 \\
8\end{array}$} & \multirow{3}{*}{$\begin{array}{l}\text { OSDI } \\
30.5 \\
25 \\
0 \\
0 \\
0\end{array}$} & \multicolumn{2}{|c|}{ FBUT (s) } & \multicolumn{2}{|c|}{ Oxford } & \multicolumn{2}{|c|}{$\begin{array}{l}\text { Schir. } \\
(\mathrm{mm})\end{array}$} & \multicolumn{2}{|c|}{ Meibum } & \multirow{3}{*}{$\begin{array}{l}\text { RCEs } \\
\text { ep. } \\
\text { Yes } \\
\text { Yes } \\
\text { No } \\
\text { No } \\
\text { No }\end{array}$} \\
\hline & & & & & \multirow{2}{*}{$\begin{array}{l}\text { R.E. } \\
4 \\
3 \\
6 \\
7 \\
8\end{array}$} & \multirow{2}{*}{$\begin{array}{l}\text { L.E. } \\
3 \\
3 \\
6 \\
8 \\
10\end{array}$} & \multirow{2}{*}{$\begin{array}{l}\text { R.E } \\
0 \\
0 \\
0 \\
0 \\
0\end{array}$} & \multirow{2}{*}{$\begin{array}{l}\text { L.E. } \\
0 \\
0 \\
0 \\
0 \\
0\end{array}$} & \multirow{2}{*}{$\begin{array}{l}\text { R.E. } \\
2 \\
5 \\
9 \\
10 \\
10\end{array}$} & \multirow{2}{*}{$\begin{array}{l}\text { L.E. } \\
1 \\
10 \\
5 \\
12 \\
10\end{array}$} & \multirow{2}{*}{$\begin{array}{l}\text { R.E. } \\
\text { N. } \\
\text { N. } \\
\text { N. } \\
\text { N. } \\
\text { N. }\end{array}$} & \multirow{2}{*}{$\begin{array}{l}\text { L.E. } \\
\text { N. } \\
\text { N. } \\
\text { N. } \\
\text { N. } \\
\text { N. }\end{array}$} & \\
\hline Patient I & $\begin{array}{l}\text { Baseline } \\
15 \text { days } \\
2 \text { months } \\
6 \text { months } \\
12 \text { months }\end{array}$ & & & & & & & & & & & & \\
\hline Patient 2 & $\begin{array}{l}\text { Baseline } \\
15 \text { days } \\
2 \text { months } \\
6 \text { months } \\
12 \text { months }\end{array}$ & Left & $\begin{array}{l}17 \\
16 \\
12 \\
10 \\
9\end{array}$ & $\begin{array}{l}33.3 \\
30.5 \\
11.3 \\
6,3 \\
4.5\end{array}$ & $\begin{array}{l}3 \\
4 \\
4 \\
8 \\
12\end{array}$ & $\begin{array}{l}2 \\
3 \\
4 \\
9 \\
11\end{array}$ & $\begin{array}{l}1 \\
1 \\
0 \\
0 \\
0\end{array}$ & $\begin{array}{l}2 \\
1 \\
0 \\
0 \\
0\end{array}$ & $\begin{array}{l}1 \\
3 \\
5 \\
7 \\
7\end{array}$ & $\begin{array}{l}0 \\
2 \\
6 \\
5 \\
6\end{array}$ & $\begin{array}{l}\text { N. } \\
\text { N. } \\
\text { N. } \\
\text { N. } \\
\text { N. }\end{array}$ & $\begin{array}{l}\text { N. } \\
\text { N. } \\
\text { N. } \\
\text { N. } \\
\text { N. }\end{array}$ & $\begin{array}{l}\text { Yes } \\
\text { Yes } \\
\text { No } \\
\text { No } \\
\text { No }\end{array}$ \\
\hline Patient 3 & $\begin{array}{l}\text { Baseline } \\
15 \text { days } \\
2 \text { month } \\
6 \text { months } \\
12 \text { months }\end{array}$ & Right & $\begin{array}{l}29 \\
27 \\
19 \\
15 \\
13\end{array}$ & $\begin{array}{l}70.8 \\
68.2 \\
34 \\
22.7 \\
18.1\end{array}$ & $\begin{array}{l}3 \\
4 \\
6 \\
6 \\
7\end{array}$ & $\begin{array}{l}3 \\
2 \\
5 \\
7 \\
7\end{array}$ & $\begin{array}{l}0 \\
0 \\
0 \\
0 \\
0\end{array}$ & $\begin{array}{l}0 \\
0 \\
0 \\
0 \\
0\end{array}$ & $\begin{array}{l}4 \\
5 \\
12 \\
12 \\
10\end{array}$ & $\begin{array}{l}6 \\
6 \\
11 \\
12 \\
11\end{array}$ & $\begin{array}{l}\text { P. } \\
P . \\
P . \\
P . \\
P .\end{array}$ & $\begin{array}{l}\text { P. } \\
P . \\
P . \\
P . \\
P .\end{array}$ & $\begin{array}{l}\text { Yes } \\
\text { Yes } \\
\text { No } \\
\text { No } \\
\text { No }\end{array}$ \\
\hline Patient 4 & $\begin{array}{l}\text { Baseline } \\
15 \text { days } \\
2 \text { months } \\
6 \text { months } \\
12 \text { months }\end{array}$ & Left & $\begin{array}{l}16 \\
14 \\
6 \\
5 \\
6\end{array}$ & $\begin{array}{l}30.2 \\
11.3 \\
0 \\
0 \\
4\end{array}$ & $\begin{array}{l}3 \\
3 \\
9 \\
12 \\
13\end{array}$ & $\begin{array}{l}2 \\
4 \\
11 \\
11 \\
12\end{array}$ & $\begin{array}{l}0 \\
0 \\
0 \\
0 \\
0\end{array}$ & $\begin{array}{l}0 \\
0 \\
0 \\
0 \\
0\end{array}$ & $\begin{array}{l}7 \\
6 \\
10 \\
12 \\
10\end{array}$ & $\begin{array}{l}7 \\
5 \\
12 \\
11 \\
12\end{array}$ & $\begin{array}{l}\mathrm{N} . \\
\mathrm{N} . \\
\mathrm{N} . \\
\mathrm{N} . \\
\mathrm{N} .\end{array}$ & $\begin{array}{l}\mathrm{N} . \\
\mathrm{N} . \\
\mathrm{N} . \\
\mathrm{N} . \\
\mathrm{N} .\end{array}$ & $\begin{array}{l}\text { Yes } \\
\text { Yes } \\
\text { No } \\
\text { No } \\
\text { No }\end{array}$ \\
\hline
\end{tabular}

Abbreviations: PEDs, persistent epithelial defects; McM, McMonnies Dry Eye Questionnaire (score); OSDI, Ocular Surface Disease Index (score); FBUT R.E/L.E., fluorescein break-up time in right eye/left eye expressed in seconds; Oxford R.E/L.E., fluorescein staining of the cornea and conjunctiva (according to the Oxford grading system) in right eye/left eye; Schir. R.E/L.E, Schirmer I test in the right/left eye (expressed in mm); Meibum R.E/L.E., meibomian secretion (meibum) after digital pressure application on the upper lid tarsus ( $N=$ Normal; $P=$ Pathologic); RCEs ep., presence of episodic RCEs symptoms (acute pain and photophobia).

At the first evaluation, all patients revealed a variable degree of DED. Among them, three patients had a mild DED (McMonnies score between 10 and 20, and OSDI score between 23 and 32) and one patient had a severe DED (McMonnies score was more than 20 and OSDI more than 33). Each patient showed a low value of FBUT in both eyes (lower than $10 \mathrm{~s}$ ) ranged from 2 to $4 \mathrm{~s}$.

The quantitative evaluation of tear production was revealed with the Schirmer I Test, which was markedly reduced in the two older patients (from 0 to $2 \mathrm{~mm}$ in both eyes), and slightly reduced in the other two patients (from 4 to $7 \mathrm{~mm}$ in both eyes).

The fluorescein staining of the cornea and conjunctiva, based on the Oxford Grading System, revealed no punctate staining in three patients and moderate staining in both eyes of the older patient.

Moreover, the meibomian secretion and the BCVA were reduced in the older patient. BVCA was 0.00 LogMAR in both eyes of three patients, and 0.10 in both eyes of the older patient.

Intraocular pressure (IOP) measured at the first check was in the range of normality (from 14 to $20 \mathrm{mmHg}$ ) (Table 1).

\section{On the 15th day after therapy}

Two weeks after the beginning of cyclosporine eye drops therapy, the clinical status of the patient tended to ameliorate in signs without subjective improvement, as follows: McMonnies Dry Eye Questionnaire score was slightly reduced in all patients, but their grade of DED gravity showed no changes (3 moderate and 1 severe). OSDI score improved in all patients. One of these was ameliorated from moderate (30.2) to normal score (11.3), and the other one was ameliorated from severe (33.3) to moderate score (30.5)

The other parameters examined (FBUT, Oxford score, Schirmer I test and meibomian secretion) showed no 
changes from the first evaluation. At this time, RCEs continued to persist in all patients (Table 2).

\section{On the 2nd month after therapy}

At the following examination, two months after the onset of cyclosporine therapy, all patient referred to feel their eyes more wet than in the past and to have a significant increase in eye comfort sensation.

We observed an important improvement of the clinical status compared to baseline. Indeed, McMonnies Dry Eye Questionnaire score was substantially improved in all patients. Two patients, compared to baseline, switched from mild to normal grade (the score changed from 15 to 8 and from 16 to 6 ). One patient ameliorated from severe to mild grade (from 29 to 19) and in one patient the McMonnies evaluation remained mild (with a score reduction from 17 to 12). Similarly to McMonnies, OSDI questionnaire revealed an improvement of the ocular discomfort in all participants. All subjects disclosed an important score reduction in OSDI value as follows: two mild patients and one severe patient raised to a normal status (from 30.5, 33.3 , and 30.5 to $0,11.3$, and 11.3 , respectively). Only one patient remained at the same severe status, although she showed a considerable reduction of her score, from 70.8 (at baseline) to 34 (subsequently).

The FBUT was increased, in relation to the baseline value, in all eyes from 1 to $9 \mathrm{~s}$ (average value was $3.5 \mathrm{~s}$ ). In addition, the Schirmer I Test showed an ameliorated outcome. All eyes revealed an increased production of tears from 3 to $7 \mathrm{~mm}$ (average value was $5 \mathrm{~mm}$ ). Fluorescein staining score was zero in all eyes (at baseline, one eye revealed a score of 1 , and another eye disclosed a score of 2). Meibomian secretion was unchanged. The episodes of RCEs were for the first time reduced in all patients.

Overall, two patients had no recurrences of ocular pain, redness, and photophobia, and two patients showed a reduction in frequency of RCEs symptoms (Table 2).

\section{On the 6th month after therapy}

On the $6^{\text {th }}$ month, all patients disclosed a stabilization of clinical pictures.

McMonnies evaluation had no significant changes, but one patient improved from a mild (12) to a normal grade (10). One patient remained in a mild grade and two participants remained in a normal score. OSDI questionnaire showed only one ameliorated patient (from mild to slight score). Other subjects showed no changes in the grading score.
FBUT, Schirmer I Test, fluorescein staining score, and meibomian secretion were analog to the previous visit.

At this examination, for the first time, all the subjects revealed a clinical improvement of DED signs and symptoms, related to the absence of new RCE episodes (Table 2).

\section{On the 12th month after therapy}

On the 12th month, no substantial differences in symptoms and signs were observed. Clinical pictures were similar to previous visit. All subjects revealed, as reported in the 6th month, the absence of RCEs symptoms (Table 2).

\section{Discussion}

The results of our case series demonstrated that the treatment with cyclosporine eye drops was useful in reducing the RCEs and the concomitant tear film dysfunctions in patients with refractory PEDs. In addition, the latter also benefited from the topical immunomodulatory therapy, with the complete healing of areas of epithelial loss. Therefore, topical cyclosporine proved here to be useful to manage complex and recalcitrant cases of ocular surface damage with associated comorbidities.

As previously reported, patients with chronic DED, if untreated, may develop ocular surface damage over time. ${ }^{9}$ Consequently, the mechanical stress due to the reduced lubrification may lead to continuous erosion and abnormal scarring in corneal and conjunctival tissue.

On the other hand, RCEs (and related PEDs) are painful conditions due to episodes of breakdown of corneal epithelium, which are characterized by a defect adhesion of epithelium to the basal membrane (E-BM). In these particular situations, the mechanical and physiological stress due to the lid movements (during blinking) may be increased by dry eye status (eg, DED) and may predispose to episodic tear or pop of epithelial blisters (Figures 1 and 2).

As is known, the treatment of PEDs is based on different therapeutic strategies, which generally lead to a variety of poorly predictable outcomes. Lubrication is usually the first step. Punctal plugs are useful to increase the retention of natural tears, which facilitates the wound healing process. However, if used in severe inflammatory disorders, they can exacerbate both the epithelial defect and DED. Soft therapeutic contact lenses are used to protect the corneal surface from mechanical trauma from the eyelids. Tarsorrhaphy is used to allow the ocular surface to heal itself. However, it is clearly reserved for noncompliant or debilitated patients, as well as for patients with lagophthalmos or exposure 
keratopathy. Oral tetracycline ( $250 \mathrm{mg}$ four times daily) and doxycycline (20-100 mg twice daily), which have anticollagenolytic properties, may aid in corneal wound healing and prevent stromal lysis. Prophylactic topical antibiotics are used to prevent the risk of infection. Again, the use of topical steroids such as loteprednol or prednisolone twice daily tends to be very helpful. In the most serious and refractory cases, the following measures are taken: human amniotic grafting, autologous serum eye drops, and scleral contact lenses. ${ }^{1}$

However, in some cases, PEDs may be non-responsive to various therapeutic strategies, and the patient may be reluctant or unable to undergo invasive or complex treatments.

Topical cyclosporine is an immunomodulatory drug with anti-inflammatory effects, which is able to inhibit IL-2 activation of lymphocytes. Accordingly, immunomodulation aims to attenuate the immune response to an adaptive rather than maladaptive level, thus promoting healing of the tissue damages. For instance, a regulatory adjustment of the immune system with cyclosporine A $1 \%$ eye drops was demonstrated to be an effective approach in the clinical picture of the ocular graft-versus-host-disease. ${ }^{16}$ In the latter context, white blood cells (T cells) of the donor's immune system which remain within the donated tissue (the graft) recognize and attack the recipient (the host) as foreign (nonself), thus determining a specific medical complication of allotransplant (in the absence of RCEs or defects of adhesion between epithelium and basal membrane).

The novelty of the present study is that topical therapy with cyclosporine A $0.05 \%$, in PED patients with DED and RCEs, has proved to be useful in improving the tear film stability and in reducing the relapse of epithelial ruptures. Again, our results showed that the recurrence rate of corneal erosions improved in parallel with the improvement of DED signs and symptoms. Therefore, the data of this work suggest that the reduction of ocular surface inflammation and the improvement of lubrication may reduce the mechanical stress that leads to a potential tear or pop of epithelial blisters. In fact, a clinically significant amelioration was disclosed in tear production, ocular surface integrity, BUT and ocular symptoms, thus indicating a potentially central role of the immunomodulatory therapy on regulation of the homeostasis of the ocular surface in these particular cases.

Specifically, ocular surface inflammation may lead $\mathrm{T}$ lymphocytes to the release of inflammatory mediators that damage the surface epithelium. ${ }^{17}$ This, in turn, results in a reduction of mucin production and in further loss of homeostasis. Conversely, less inflammation implies an improvement in the quality of the epithelium, which leads to an increased reflex tearing with a greater supply of nutrients, growth factors and protective substances for the ocular surface epithelia (eg, secretory IgA, lysozyme, lactoferrin, or hepatocyte growth factor and epidermal growth factor that are essentials for the maintenance of the epithelium). In our analysis, this was reflected in the better Schirmer test and fluorescein staining scores. Similarly, an improvement in the interface between the aqueous tear layer and the hydrophobic epithelium due to a better mucin production would explain the increase in BUT results. ${ }^{18}$

Thus, we believe that cyclosporine eye drop is a promising therapeutic strategy, mainly because it was very effective in stabilizing the health status of the ocular surface in absence of any systemic or local side effect. Clearly, this aspect was very important for our patients, since PEDs and associated comorbidities may be difficult to treat and can require long-term therapy. Interestingly, some clinical trials demonstrated that topical cyclosporine therapy can be well tolerated for up to 3 years without major adverse effects. ${ }^{19,20}$ In this sense, it should be further taken into consideration and studied as primary therapy, or in addition to initial therapy to maximize its effectiveness, and in patients with elevated risk of relapse of epithelial ruptures.

In conclusion, despite the low sample size due to the narrow inclusion criteria for this study, topical cyclosporine medication can be considered an interesting, safe and effective treatment option in the management of refractory PEDs with associated ocular comorbidities.

\section{Acknowledgments}

The authors wish to express their gratitude to Luigi Ventura for proofreading the article. This research received no specific grant from any funding agency in the public, commercial or not-for-profit sectors. The authors have no financial or proprietary interest in products, methods, or material used in this research.

\section{Disclosure}

The authors report no conflicts of interest in this work.

\section{References}

1. Rolando M, Zierhut M. The ocular surface and tear film and their dysfunction in dry eye disease. Surv Ophthalmol. 2001;45:S203-S210.

2. Napoli PE, Nioi M, Iovino C, Sanna R, d'Aloja E, Fossarello M. Ocular surface and respiratory tract damages from occupational, subchronic exposure to fluorspar: case report and other considerations. Int Ophthalmol. 2019;39(5):1175-8. 
3. Katzman LR, Jeng BH. Management strategies for persistent epithelial defects of the cornea. Saudi J Ophthalmol. 2014;28(3):168-172. doi:10.1016/j.sjopt.2014.06.011

4. Napoli PE, Nioi M, d'Aloja E, Fossarello M. The bull's eye pattern of the tear film in humans during visual fixation on en-face optical coherence tomography. Sci Rep. 2019;9(1):1413. doi:10.1038/s41598-018-38260-5

5. Bron AJ, Evans VE, Smith JA. Grading of corneal and conjunctival staining in the context of other dry eye tests. Cornea. 2003;22 (7):640-650.

6. Dahlgren, M.A., Dhaliwal A, Huang AJ. Persistent epithelial defects. In: Albert D, Miller J, Azar D, Blodi B, editors. Albert \& Jakobiec's Principles and Practice of Ophthalmology. $3^{\text {rd }}$ ed. Philadelphia, Pennsylvania, USA: Elsevier; 2008:749-759. ISBN 9780323313575.

7. Chen YT, Huang CW, Huang FC, Tseng SY, Tseng SH. The cleavage plane of corneal epithelial adhesion complex in traumatic recurrent corneal erosion. Mol Vis. 2006;12:196-204.

8. Craig JP, Nelson JD, Azar DT, et al. TFOS DEWS II report executive summary. Ocul Surf. 2017;15:802-812. doi:10.1016/j.jtos.2017.08.003

9. Marshall LL, Roach JM. Treatment of dry eye disease. Consult Pharm. 2016;31:96-106. doi:10.4140/TCP.n.2016.96

10. Napoli PE, Coronella F, Satta GM, Galantuomo MS, Fossarello M, Huang J. Evaluation of the adhesive properties of the cornea by means of optical coherence tomography in patients with meibomian gland dysfunction and lacrimal tear deficiency. PLoS One. 2014;9 (12):e115762. doi:10.1371/journal.pone.0115762

11. Nioi M, Napoli PE, Demontis R, Locci E, Fossarello M, d'Aloja E. Morphological analysis of corneal findings modifications after death: a preliminary OCT study on an animal model. Exp Eye Res. 2018;169:20-27. doi:10.1016/j.exer.2018.01.013

12. Napoli PE, Satta GM, Coronella F, Fossarello M. Spectral-domain optical coherence tomography study on dynamic changes of human tears after instillation of artificial tears. Invest Ophthalmol Vis Sci. 2014;55(7):4533-4540. doi:10.1167/iovs.14-14666
13. Wise RJ, Sobel RK, Allen RC. Meibography: a review of techniques and technologies. Saudi $J$ Ophthalmol. 2012;26:349-356. doi:10.1016/j.sjopt.2012.08.007

14. Shimazaki J, Sakata M, Tsubota K. Ocular surface changes and discomfort in patients with meibomian gland dysfunction. Arch Ophthalmol. 1995;113:1266-1270.

15. Napoli PE, Coronella F, Satta GM, Iovino C, Sanna R, Fossarello M. A simple novel technique of infrared meibography by means of spectral-domain optical coherence tomography: a cross-sectional clinical study. PLoS One. 2016;11(10):e0165558. doi:10.1371/journal.pone. 0165558

16. Kiang E, Tesavibul N, Yee R, Kellaway J, Przepiorka D. The use of topical cyclosporin A in ocular graft-versus-host-disease. Bone Marrow Transplant. 1998;22(2):147. doi:10.1038/sj.bmt.1701300

17. Wan KH, Li J, Alvin LY. Efficacy and safety of topical $0.05 \%$ cyclosporine eye drops in the treatment of dry eye syndrome: a systematic review and meta-analysis. Ocul Surf. 2015;13 (3):213-225. doi:10.1016/j.jtos.2014.12.006

18. Napoli PE, Coronella F, Satta GM, Zucca IA, Fossarello M. A novel OCT technique to measure in vivo the corneal adhesiveness for sodium carboxymethylcellulose in humans and its validity in the diagnosis of dry eye. Invest Ophthalmol Vis Sci. 2014;55 (5):3179-3185. doi:10.1167/iovs.13-13613

19. Barber LD, Pflugfelder SC, Tauber J, Foulks GN. Phase III safety evaluation of cyclosporine $0.1 \%$ ophthalmic emulsion administered twice daily to dry eye disease patients for up to 3 years. Ophthalmology. 2005;112:1790-1794. doi:10.1016/j. ophtha.2005.05.013

20. Stevenson D, Tauber J, Reis BL. Efficacy and safety of cyclosporin A ophthalmic emulsion in the treatment of moderate-to-severe dry eye disease: a dose-ranging, randomized trial. Ophthalmology. 2000;107:967-974.

\section{Publish your work in this journal}

Drug Design, Development and Therapy is an international, peerreviewed open-access journal that spans the spectrum of drug design and development through to clinical applications. Clinical outcomes, patient safety, and programs for the development and effective, safe, and sustained use of medicines are a feature of the journal, which has also been accepted for indexing on PubMed Central. The manuscript management system is completely online and includes a very quick and fair peer-review system, which is all easy to use. Visit http://www. dovepress.com/testimonials.php to read real quotes from published authors. 